\title{
Design of Dynamic Monitoring System for Maize Growth Information Based on Internet of Things
}

\author{
Bi Xiaoming ${ }^{1,}$, Tong Jian ${ }^{1, b}$ \\ ${ }^{1}$ Wenzhou Vocational College of Science and Technology, Wenzhou Zhejiang, 325006, China \\ a1927210737@qq.com, b63619631@qq.com
}

Keywords: Internet of things; maize growth information; dynamic monitoring system; design.

\begin{abstract}
In view of the design of dynamic monitoring system for maize growth information and the related technology of Internet of things, the principle and system composition of the design of maize growth information dynamic monitoring system are analyzed on the basis of a brief description of the growth process of maize. Finally, the feasibility of the design of the system is analyzed in the field through the field experiment. Finally, through in-depth analysis, it is concluded that the dynamic monitoring system of maize growth information based on the Internet of things can effectively guarantee the true and effective results of corn growth information measurement results, and hope to help improve the yield of maize in China.
\end{abstract}

\section{Introduction}

The information of maize growth can be divided into two categories. One kind of appearance information, including plant height and plant type, can be obtained by direct observation and recording, which mainly reflects the health status of maize growth. The other is intrinsic information, including all kinds of physiological locations of maize growth, which can be measured by corresponding instruments and special experimental methods. At present, the dynamic monitoring of maize growth information in China is completed manually. Therefore, the monitoring results are closely related to the quality of the monitoring staff, and it is difficult to truly and accurately reflect the growth information of maize. It's particularly important to explore new monitoring system based on such a basis. Based on the technology of Internet of things, a dynamic monitoring system of maize growth information is designed in this paper.

\section{The Growth Process of Maize}

The growth process of maize can be generally divided into the following processes.

\subsection{Growth period}

The birth period refers to the days of maize from sowing to maturity, the variety of maize, the different sowing time, different light and different temperature, and the number of days of its growth period is not the same. Under normal circumstances, the early maturity of maize varieties is relatively short when light is sufficient and the temperature is high. The growth stages of maize can be divided into three stages: seeding, seedling emergence, three leaf, seven leaf, jointing, heading, flowering, filling, milk ripening and maturity.

\subsection{Seedling stage}

The seedling stage of maize refers to the period of emergence and elongation of maize, and also the main period for maize to absorb nutrition. About $60 \%$ of the maize seedlings can grow to $2 \mathrm{~cm}$.

\subsection{Panicle stage}

Corn refers to the maize from jointing to heading period, this period is the most vigorous stage of corn growth. 


\subsection{Flower grain period}

The flowering stage of maize refers to the period from heading to fruiting, and this stage is mainly based on reproductive growth, and it is also a stage of maize grain formation.

\section{The Design Principle of Dynamic Monitoring System of Maize Growth Information Based on Internet of Things}

\subsection{The design principle of corn growth information dynamic monitoring system}

Based on the Internet of Things technology, a corn growth information collection device, an information transmission system, and an intelligent analysis system are established so as to realize dynamic monitoring and remote control of corn growth information. Then through the use of appropriate radio frequency identification technology, spectral analysis technology, etc. to scientifically and reasonably monitor corn growth morphology, yield, physiological indicators, pests and other related data. Then scientifically and reasonably analyzing the corresponding data and information, all information on corn growth can be obtained. Finally, through adjustment and transformation, a good environment for the growth of corn is created to achieve the goal of increasing corn production, shortening the growth cycle, and saving cost. .

\subsection{Overall design}

In recent years, China's Internet of Things technology has developed rapidly and has become more widely used in agricultural production. An information-based dynamic monitoring system integrating corn growth information data collection, information transmission, and data processing has been designed. The main task of the data acquisition system is to sense the corn growth information and maintain the system's continuous and stable operation. The information transmission system refers to the transmission of related information and numbers through the Internet of Things technology and 4G network technology. The data processing system can perform reasonable statistics and analysis on corn growth information [1]. In short, the main support for the dynamic monitoring system of corn growth information based on the Internet of Things is the plant factory. Because of the main mode of agricultural development at present in plant factories, the internal lighting time and temperature are all controllable and will not be influenced by external natural factors, thus creating a good environment for corn growth.

\subsection{The composition of the system}

The Internet-based corn growth information dynamic monitoring system is mainly composed of the following four parts:

First, a video data acquisition device. The key technology of the device is computer vision technology. For example, the content of chlorophyll and total nitrogen in corn growth can be measured by a spectrometer, and the nutrient status of corn during the growth process can be controlled in time. The content of chlorophyll is the dynamic detection of corn growth information. The main indicators, according to the specific value of monitoring to develop a corresponding solution.

Second, the signal transmission device, the main means of transmitting information and data between the device's video information acquisition device and the data processor. The COFDM modulation technology is mainly used to ensure the continuity and reliability of corn growth information transmission.

Third, the data processing server is mainly responsible for receiving and storing corn growth information data, and analyzing and processing related information transmitted to the relevant technical personnel and experts for advanced analysis of corn growth information. Mainly through the serial port under the CE environment of the computer system to improve the efficiency of data processing.

Fourth, the background management system, the main role of this system to actively receive relevant information and data, and then according to the processing server feedback analysis results 
and expert decision-making on the problems of corn growth to develop appropriate solutions to ensure the normal growth of corn.

\section{Feasibility Analysis of System Design}

\subsection{Corn field trials}

In 2016, a plant-based plant was used to verify the dynamic monitoring system of corn growth information based on the Internet of Things. The test soil was the local soil of sublimation maize. The test results showed that the content of soil organic matter was $26.4 \mathrm{~g} / \mathrm{kg}$. The nitrogen content was $132 \mathrm{mg} / \mathrm{kg}$, the phosphorus content was $12.4 \mathrm{mg} / \mathrm{kg}$, the potassium content was $146 \mathrm{mg} / \mathrm{kg}$, and the soil pH was 5.34 [2]. The experimental corn seeds were Nonghua 101, Weike 702, Chuanglian 808, and Longping 208. These four kinds of seeds were all purchased from the local farmers' market, so they were representative. These four kinds of corn seeds are currently the most cultivated corn products in China, and their planting area is very large.

\subsection{Test process}

Corn was sown in late April and transplanted in late July. The four corn varieties were planted in a random arrangement. The row control of corn is controlled between $20 \sim 25 \mathrm{~cm}$, and the spacing between the rows is controlled between $15 \sim 20 \mathrm{~cm}$. Regardless of corn water and fertilizer irrigation or pest and disease prevention are carried out in accordance with the contents of the Internet of Things monitoring system, and choose the height of corn growth, spike length, chlorophyll content for the monitoring of the Internet of Things monitoring system [3]. Thirty maize plants were randomly selected to measure the height of corn growth, the length of spikes, and the monitoring of the chlorophyll content in the corn using the SPAD502 chlorophyll meter. In order to improve the efficiency of the experiment, the heading of maize was selected for testing, and the relevant data was statistically analyzed on the SSPS17.0 computer system software, and the significance of the growth information of the IOT-detection system and the traditional manual detection was tested using the t-value. .

\subsection{Test results}

The test results indicate that the heights of the four maize varieties adopting the Internet of Things monitoring system are generally higher than those of traditional measurements, and there is an extremely significant level between the two. The main reason is that corn is gradually maturing at the heading stage, and its weight increases, which inevitably limits the height of corn. The height of the artificial measurement from the ground to the top of the corn, and the height monitored by the Internet of Things monitoring technology system is the height of the corn in the natural state, so there are certain differences in the two methods of measuring the height of the plant. However, the plant height of the four corn varieties is basically the same, which fully verifies the reliability of the Internet of Things monitoring system in monitoring plant height of corn growth information [4]. The corn traits obtained by the two methods are shown in Table 1:

Table 1 Maize traits obtained by two methods

\begin{tabular}{|l|l|l|l|l|l|l|}
\hline \multirow{2}{*}{ Corn varieties } & \multicolumn{2}{|l|}{ Plant height/cm } & \multicolumn{2}{l|}{ Spike length/cm } & \multicolumn{2}{l|}{ Chlorophyll content (SPAD) } \\
\cline { 2 - 7 } & Artificial & IoT & Artificial & IoT & Artificial & IoT \\
\hline Nonghua 101 & 248 & 245 & 26 & 27 & 40.4 & 38.7 \\
\hline Weike 702 & 246 & 243 & 31 & 30 & 44.6 & 42.1 \\
\hline Chuanglian 808 & 247 & 244 & 29 & 28 & 43.4 & 42.6 \\
\hline Longping 208 & 246 & 245 & 32 & 27 & 45.2 & 43.4 \\
\hline
\end{tabular}

It can be clearly seen from Table 1 that the lengths of the four corn cultivars derived from the monitoring of corn growth information and the manual measurement of corn growth information by the Internet of Things are basically the same, but the major difference in Longping 208 is due to the fact that a few varieties emerged. The phenomenon of empty spikes has led to the lack of effective 
identification of the Internet of Things. This point also fully shows that monitoring the corn growth information by the Internet of Things can effectively monitor the length of eared corn, but further optimization of setting parameters is needed to improve the ability to identify earless corn. The corn chlorophyll content obtained by the two monitoring methods is basically the same. It can be seen that the monitoring and monitoring system of the Internet of Things is efficient and reliable for the dynamic monitoring of these four types of corn growth information. However, relevant parameters need to be optimized. After these data are entered into the background management, relevant instructions and measures can be obtained, so as to maximize the Increase corn production.

\section{Summary}

In summary, based on the Internet of Things video data acquisition system, spectrum analysis system, and information transmission technology of the corn growth information dynamic monitoring system, it is a new type of monitoring technology, and through the test comparison shows that this technology and traditional manual measurement Compared with technology, not only has the efficiency of measurement been improved, but the accuracy of measurement has also been greatly improved, and it is worthy of promotion

\section{Acknowledgment}

The paper is one of the results of 2017 Wenzhou Science and Technology Plan Project "Study on the Construction of Stereoscopic Production Control System for Strawberry Based on Internet of Things" (No.N20170009), and is one of the results of Zhejiang University Student Science and Technology Innovation Project (Zhejiang Xinmiao Talents Project) "The Design of Dynamic Monitoring System for Corn Growth Information Based on Internet of Things"

\section{References}

[1] zheng yanbin, li na, andyu. Based on the maize illumination inverse optical line tracking algorithm based on the binary-inclosed box [J/OL]. Computer application research, 2018, (07):1-2(2017-07-27).

[2] Qin Peiliang jaew, Chen wen, liu tao, cheng-ming sun. Crop water intelligent management research progress [J/OL]. Crops, 2017, (5): 1-6 (2017-09-29).

[3] deng xiaolu, wang pei, ma ning, liu hui. Design and implementation of the early warning system of the cold ground corn spot disease based on the Internet of things [J]. Journal of agricultural mechanization in China, 2016, 37(07):166-170.

[4] Dong shujuan. Design of dynamic monitoring system for rice growth information -- based on Internet of things technology [J]. Agricultural mechanization research, 2013, 39(08):216-219. 